\title{
A Novel Kumada Coupling Reaction to access Cyclic (2-Azaallyl)stannanes. Cycloadditions of Cyclic Nonstabilized 2-Azaallyllithium Species derived from the Cyclic (2-Azaallyl)stannanes.
}

\author{
Douglas M. Mans and William H. Pearson* *
}

Department of Chemistry, University of Michigan, Ann Arbor, MI 48109-1055, USA

Email: wpearson@berryassoc.com

$†$ Current Address: Berry and Associates, Inc., 2434 Bishop Circle East, Dexter, MI, 48130 USA.

\section{Supporting Information}

\section{Table of Contents}

Experimental procedures for $\mathbf{2 b}, \mathbf{1 9 - 2 2}, \mathbf{2 4}, \mathbf{3 4 - 3 6}, \mathbf{4 5 - 5 2}, \mathbf{5 5 - 5 8}$.

pages: S2-S21

${ }^{1} \mathrm{H} /{ }^{13} \mathrm{C}$ NMR spectra for compounds $\mathbf{1 9 - 2 2 ,}$ 24, 34-36, 45-52, 55-58 pages: S22-S72 


\section{EXPERIMENTAL}

General Methods. All reactions were performed under an atmosphere of dry nitrogen or argon in flame-dried glassware equipped with tightly fitting rubber septa. All syringes and needles were oven-dried and cooled in a dessicator prior to use. Reactions at $0{ }^{\circ} \mathrm{C}$ were carried out in an ice/water bath. Reactions at $-78{ }^{\circ} \mathrm{C}$ were carried out in a dry ice/acetone bath. Solvents were freshly prepared before use. Benzene, toluene, methylene chloride $\left(\mathrm{CH}_{2} \mathrm{Cl}_{2}\right)$, pyridine, triethylamine and diisopropylamine were distilled from calcium hydride. Tetrahydrofuran (THF) and diethyl ether were distilled from sodium/benzophenone ketyl. Dimethylformamide (DMF) was distilled from barium oxide under reduced pressure. $n$-Butyllithium was titrated with diphenylacetic acid prior to use.

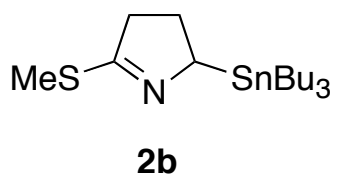

2-Thiomethoxy-5-(tri-n-butylstannyl)-1-pyrroline (2b): Stannyl lactam 11 ${ }^{\text {1b }}(523 \mathrm{mg}$, $1.4 \mathrm{mmol}$ ) and Lawesson's reagent (274 mg, $0.68 \mathrm{mmol})$ were combined in THF (10 mL) and heated under reflux. After $30 \mathrm{~min}$, the reaction was diluted with ethyl acetate, washed with $10 \% \mathrm{NaOH}(\mathrm{aq})$, dried $\left(\mathrm{MgSO}_{4}\right)$, filtered, and concentrated to provide a thick oil. Chromatography (0-5\% ethyl acetate/hexanes gradient) afforded $330 \mathrm{mg}(60 \%)$ of the stannyl thiolactam as a nearly colorless oil. $\mathrm{R}_{f}=0.4$ (20\% ethyl acteate/hexanes); ${ }^{1} \mathrm{H}$ NMR (200 MHz, $\left.\mathrm{CDCl}_{3}\right) \delta 9.08(\mathrm{br} \mathrm{s}, 1 \mathrm{H}), 3.76(\mathrm{t}, 1 \mathrm{H}, J=7.9 \mathrm{~Hz}), 3.08-2.72(\mathrm{~m}, 2$ H), 2.60-2.43 (m, $1 \mathrm{H}), 2.32-2.12(\mathrm{~m}, 1 \mathrm{H}), 1.60-1.40(\mathrm{~m}, 6 \mathrm{H}), 1.40-1.24(\mathrm{~m}, 6 \mathrm{H}), 1.08$ $0.89(\mathrm{~m}, 15 \mathrm{H}) ;{ }^{13} \mathrm{C} \mathrm{NMR}\left(90 \mathrm{MHz}, \mathrm{CDCl}_{3}\right) \delta 201.6,50.3,44.1,29.0(\mathrm{~J}=21 \mathrm{~Hz}), 28.6$, $27.3(J=54 \mathrm{~Hz}), 13.6,9.1(J=310 \mathrm{~Hz})$; IR (neat) $3134(\mathrm{br}, \mathrm{m}), 1524(\mathrm{~s}) \mathrm{cm}^{-1}$; MS (CI, 
$\left.\mathrm{CH}_{4}\right) \mathrm{m} / z$ (rel int) 392 (100), 291 (26), 118 (21), 102 (66), 84 (67); HRMS (CI, $\mathrm{CH}_{4}$ ) calcd for $\mathrm{C}_{16} \mathrm{H}_{34} \mathrm{NSSn}(\mathrm{M}+\mathrm{H})^{+}$392.1434; found 392.1414.

The stannyl thiolactam $(340 \mathrm{mg}, 0.87 \mathrm{mmol})$ and dimethyl sulfate $(90 \mu \mathrm{L}, 0.95$ mmol) were mixed neat. After $12 \mathrm{~h}$, the reaction was diluted with ether, washed with $10 \% \mathrm{~K}_{2} \mathrm{CO}_{3}(\mathrm{aq})$, dried $\left(\mathrm{Na}_{2} \mathrm{SO}_{4}\right)$, filtered, and concentrated to provide an oil of mass 323 mg $(92 \%)$. The product was used without further purification. Data for $(\mathbf{2 b}):{ }^{1} \mathrm{H}$ NMR $\left(360 \mathrm{MHz}, \mathrm{CDCl}_{3}\right) \delta 4.20\left(\mathrm{dt}, 1 \mathrm{H}, J=8.7,2.0 \mathrm{~Hz},{ }^{2} J\left({ }^{119 / 117} \mathrm{Sn}-{ }^{1} \mathrm{H}\right)=35.9 \mathrm{~Hz}\right), 2.63(\mathrm{ddd}$, $1 \mathrm{H}, J=16.0,10.0,3.1 \mathrm{~Hz}), 2.50-2.20(\mathrm{~m}, 5 \mathrm{H}), 2.14(\mathrm{ddt}, 1 \mathrm{H}, J=12.1,9.4,2.2 \mathrm{~Hz})$, 1.53-1.45 (m, $6 \mathrm{H}), 1.37-1.25$ (m, $6 \mathrm{H}), 0.95-0.80(\mathrm{~m}, 15 \mathrm{H}) ;{ }^{13} \mathrm{C}$ NMR (90 MHz, $\mathrm{CDCl}_{3}$ ) $\delta$ 165.0, 63.7, 38.4, 29.4, $29.1(J=20 \mathrm{~Hz}), 27.4(J=52 \mathrm{~Hz}), 13.7,9.3$; IR (neat) $1741(\mathrm{w})$ $\mathrm{cm}^{-1}$; MS (CI, $\left.\mathrm{NH}_{3}\right) \mathrm{m} / z$ (rel int) 406 (7, [M+H]), 291 (33), 235 (75), 233 (58), 179 (100), 114 (83), 84 (78); HRMS (CI, $\mathrm{NH}_{3}$ ) calcd for $\mathrm{C}_{17} \mathrm{H}_{36} \mathrm{NSSn}[\mathrm{M}+\mathrm{H}]^{+}$406.1590; found 406.1588 .

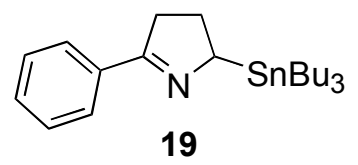

5-Phenyl-2-(tri- $\boldsymbol{n}$-butylstannyl)-3,4-dihydro-2H-pyrrole (19): To a flask containing stannane $\mathbf{2 b}$ (50.0 $\mathrm{mg}, 0.12 \mathrm{mmol})$ and [1,1'bis(diphenylphosphino)ferrocene]dichloropalladium (II) $(2.50 \mathrm{mg}, 0.0031 \mathrm{mmol})$ in toluene $(1.3 \mathrm{~mL})$ was added phenylmagnesium bromide in a dropwise fashion $(62.0 \mu \mathrm{L}$ of a $3.0 \mathrm{M}$ solution in $\left.\mathrm{Et}_{2} \mathrm{O}, 0.18 \mathrm{mmol}\right)$. After $4 \mathrm{~h}$, the mixture was diluted with water and extracted with ether (3x). The combined organic phases were dried $\left(\mathrm{Na}_{2} \mathrm{SO}_{4}\right)$ and concentrated. Chromatography (5\% ethyl acetate/hexanes) yielded $29.0 \mathrm{mg}(55 \%)$ of $\mathbf{1 9}$ as an oil. Data for 19: $\mathrm{R}_{f}=0.82\left(20 \%\right.$ ethyl acetate/hexanes); ${ }^{1} \mathrm{H}$ NMR $(500 \mathrm{MHz}$, 
$\left.\mathrm{CDCl}_{3}\right) \delta 7.80(\mathrm{dd}, 2 \mathrm{H}, J=8.5,2.0 \mathrm{~Hz}), 7.41-7.36(\mathrm{~m}, 3 \mathrm{H}), 4.48(\mathrm{ddd}, 1 \mathrm{H}, J=8.5,8.5$, $2.0 \mathrm{~Hz}$ ), 3.11 (ddd, $1 \mathrm{H}, J=16.0,10.0,2.0 \mathrm{~Hz}$ ), 2.65 (dddd, $1 \mathrm{H}, J=17.0,14.0,10.0,2.5$ Hz), 2.52 (ddt, $1 \mathrm{H}, J=12.5,10.5,9.0 \mathrm{~Hz}), 2.23(\mathrm{ddt}, 1 \mathrm{H}, J=12.5,4.0,2.0 \mathrm{~Hz}), 1.55-$ 1.49 (m, $6 \mathrm{H}), 1.36-1.29(\mathrm{~m}, 6 \mathrm{H}), 0.91(\mathrm{dd}, 6 \mathrm{H}, J=8.0 \mathrm{~Hz}), 0.88(\mathrm{t}, 9 \mathrm{H}, J=7.5 \mathrm{~Hz})$;

${ }^{13} \mathrm{C} \mathrm{NMR}\left(125 \mathrm{MHz}, \mathrm{CDCl}_{3}\right) \delta 166.2,135.2,129.2,128.2,127.0,65.0,34.5,29.2$ $\left(J_{\mathrm{Sn}}{ }^{117 / 119}=10.5 \mathrm{~Hz}\right), 28.7,27.4\left(J_{\mathrm{Sn}}{ }^{117 / 119}=26.4 \mathrm{~Hz}\right), 13.7,9.6\left(J_{\mathrm{Sn}}{ }^{117 / 119}=147.5 \mathrm{~Hz}\right) ; \mathrm{IR}$ $\left(\mathrm{CHCl}_{3}\right) 1596$ (w), 1569 (w), 1494 (w) $\mathrm{cm}^{-1}$; MS (EI, $70 \mathrm{eV}$ ) $\mathrm{m} / \mathrm{z}$ (rel int) $435.0\left(3, \mathrm{M}^{+}\right)$ 378.0 (9), 322.0 (29), 291.0 (32), 268.9 (78), 143.0 (100); HRMS (EI, 70 eV) calcd for $\mathrm{C}_{22} \mathrm{H}_{37} \mathrm{NSn}\left(\mathrm{M}^{+}\right)$435.1948; found 435.1935 .

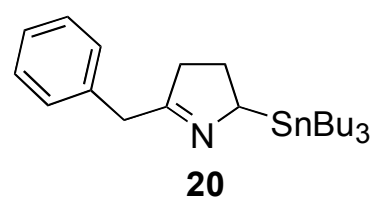

5-Benzyl-2-(tri- $n$-butylstannyl)-3,4-dihydro-2H -pyrrole (20): A deoxygenated solution of benzylzinc bromide ( $4.0 \mathrm{mmol}$ of a $0.5 \mathrm{M}$ solution in THF, $2.0 \mathrm{mmol}$ ) was transferred via cannula to a flask containing tetrakis(triphenylphosphine)palladium (0) $(0.012 \mathrm{~g}, 0.01 \mathrm{mmol})$ and stannane $2 \mathbf{b}(0.388 \mathrm{~g}, 0.95 \mathrm{mmol})$ under argon. The mixture was heated to $60{ }^{\circ} \mathrm{C}$ for $24 \mathrm{~h}$ then cooled to room temperature and diluted with ethyl acetate. The combined organic phases were washed with $10 \%$ aqueous sodium bicarbonate. Back extracted the aqueous layer with ethyl acetate (2x). Organics were combined, dried $\left(\mathrm{Na}_{2} \mathrm{SO}_{4}\right)$ and concentrated. Chromatography (0-20\% ethyl acetate/hexanes gradient) yielded $0.193 \mathrm{~g},(45 \%)$ of the title compound $\mathbf{2 0}$ as a clear yellow oil. Data for 20: $\mathrm{R}_{f}=0.24$ (20\% ethyl acetate/hexanes); ${ }^{1} \mathrm{H}$ NMR $(500 \mathrm{MHz}$, $\left.\mathrm{CDCl}_{3}\right) \delta$ 7.32-7.20 (m, $\left.5 \mathrm{H}\right), 4.15(\mathrm{~d}, 1 \mathrm{H}, J=9.0 \mathrm{~Hz}), 3.66(\mathrm{bs}, 2 \mathrm{H}), 2.45-2.39(\mathrm{~m}, 1$ H), 2.22-1.94 (m, $3 \mathrm{H}), 1.53-1.44(\mathrm{~m}, 6 \mathrm{H}), 1.29$ (quint, $6 \mathrm{H}, J=7.5 \mathrm{~Hz}), 0.88(\mathrm{t}, 9 \mathrm{H}, J=$ 
$7.5 \mathrm{~Hz}), 0.85-0.76(\mathrm{~m}, 6 \mathrm{H}) ;{ }^{13} \mathrm{C} \mathrm{NMR}\left(100 \mathrm{MHz}, \mathrm{CDCl}_{3}\right) \delta 170.1,138.0,129.2,128.7$, $126.6,63.3,40.9,36.4,29.4\left(J_{\mathrm{Sn}^{1}}{ }^{17 / 119}=10.5 \mathrm{~Hz}\right), 28.8,27.7\left(J_{\mathrm{Sn}}{ }^{117 / 119}=26.8 \mathrm{~Hz}\right), 13.9,9.3$ $\left(J_{\mathrm{Sn}}{ }^{117 / 119}=151.8 \mathrm{~Hz}\right) ; \mathrm{IR}\left(\mathrm{CHCl}_{3}\right) 1622(\mathrm{w}), 1602(\mathrm{w}), 1495(\mathrm{w}) \mathrm{cm}^{-1}$; MS (DCI w/ $\left.\mathrm{NH}_{3}\right)$ $m / z$ (rel int) $450.5\left(60,[\mathrm{M}+\mathrm{H}]^{+}\right), 392.4$ (35), 336.3 (19), 158.2 (52), 91.1 (100); HRMS (DCI w/ $\mathrm{NH}_{3}$ ) calcd for $\mathrm{C}_{23} \mathrm{H}_{40} \mathrm{NSn}\left([\mathrm{M}+\mathrm{H}]^{+}\right)$450.2183; found 450.2177.

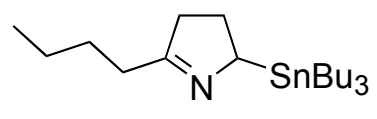

21

5-Butyl-2-(tri-n-butylstannyl)-3,4-dihydro-2H -pyrrole (21): $n$-Butylmagnesium chloride ( $81 \mu \mathrm{L}$ of a $2.0 \mathrm{M}$ solution in THF, $0.16 \mathrm{mmol}$ ) was added in a dropwise fashion to a mixture of [1,3-bis(diphenylphosphinopropane)]dichloronickel (II) (3.6 mg, 0.0066 $\mathrm{mmol})$ and toluene $(1.0 \mathrm{~mL})$ placed in a water bath. After $5 \mathrm{~min}$. a solution of stannane 2b $(60 \mathrm{mg}, 0.15 \mathrm{mmol})$ and toluene $(0.5 \mathrm{~mL})$ was added in a dropwise fashion. The reaction was allowed to stir for $15 \mathrm{~min}$. and then was diluted with water and extracted with hexanes $(4 \mathrm{x})$. The combined organic phases were dried $\left(\mathrm{Na}_{2} \mathrm{SO}_{4}\right)$ and concentrated. Chromatography (5\% ethyl acetate/hexanes) yielded $50 \mathrm{mg}(80 \%)$ of $\mathbf{2 1}$ as a clear slightly brown oil. Data for 21: $\mathrm{R}_{f}=0.4$ (20\% ethyl acetate/hexanes); ${ }^{1} \mathrm{H}$ NMR (500 $\left.\mathrm{MHz}, \mathrm{CDCl}_{3}\right) \delta 4.12\left(\mathrm{dt}, 1 \mathrm{H}, J=9.0,1.8 \mathrm{~Hz}, J_{\mathrm{Sn}}{ }^{117 / 119}=19.0 \mathrm{~Hz}\right), 2.52(\mathrm{ddd}, 1 \mathrm{H}, J=$ 17.0, 10.0, 3.0 Hz), 2.37-2.25 (m, $3 \mathrm{H}), 2.17-2.09$ (m, $1 \mathrm{H}), 2.03$ (ddt, $1 \mathrm{H}, J=12.0,5.0$, $2.5 \mathrm{~Hz}), 1.58-1.46(\mathrm{~m}, 8 \mathrm{H}), 1.39-1.27(\mathrm{~m}, 8 \mathrm{H}), 0.94-0.85(\mathrm{~m}, 18 \mathrm{H}) ;{ }^{13} \mathrm{C}$ NMR $(100$ $\left.\mathrm{MHz}, \mathrm{CDCl}_{3}\right), \delta 171.9,63.1,37.1,29.4,29.3\left(J_{\mathrm{Sn}}{ }^{117 / 119}=10.6 \mathrm{~Hz}\right), 28.4,27.7\left(J_{\mathrm{Sn}}{ }^{117 / 119}=\right.$ 27.3), 23.0, 14.1, 13.9, $9.3\left(J_{\mathrm{Sn}}{ }^{117 / 119}=147.6 \mathrm{~Hz}\right)$; IR $\left(\mathrm{CHCl}_{3}\right) 1624(\mathrm{w}) \mathrm{cm}^{-1}$; MS (CI w/ 
$\left.\mathrm{NH}_{3}\right) \mathrm{m} / z$ (rel int) $416.2\left(100,[\mathrm{M}+\mathrm{H}]^{+}\right), 308.1$ (3), 124.1 (49); HRMS (CI w/ $\mathrm{NH}_{3}$ ) calcd for $\mathrm{C}_{20} \mathrm{H}_{42} \mathrm{NSn}\left([\mathrm{M}+\mathrm{H}]^{+}\right)$416.2339; found 416.2326.

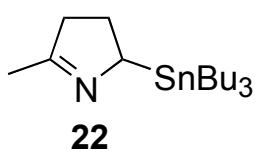

5-Methyl-2-(tri- $\boldsymbol{n}$-butylstannyl)-3,4-dihydro-2H -pyrrole (22): Methylmagnesium chloride (64 $\mu \mathrm{L}$ of a $2.57 \mathrm{M}$ solution in THF, $0.165 \mathrm{mmol}$ ) was added in a dropwise fashion to a mixture of [1,3-bis(diphenylphosphinopropane)]dichloronickel (II) (4.0 mg, $0.0075 \mathrm{mmol})$ and toluene $(1.0 \mathrm{~mL})$ placed in a water bath. After $5 \mathrm{~min}$. a solution of stannane $\mathbf{2 b}(60 \mathrm{mg}, 0.15 \mathrm{mmol})$ and toluene $(0.5 \mathrm{~mL})$ was added in a dropwise fashion. After 15 min the reaction mixture was diluted with water and extracted with hexanes (4x). The combined organic phases were dried $\left(\mathrm{Na}_{2} \mathrm{SO}_{4}\right)$ and concentrated. Chromatography (0-5\% ethyl acetate/hexanes gradient) yielded $41.8 \mathrm{mg}$ (75\%) of $\mathbf{2 2}$ as a clear yellowish oil. Data for 22: $\mathrm{R}_{f}=0.2$ (20\% ethyl acetate/hexanes); ${ }^{1} \mathrm{H}$ NMR (400 $\left.\mathrm{MHz}, \mathrm{CDCl}_{3}\right) \delta 4.11(\mathrm{dd}, 1 \mathrm{H}, J=8.8,2.0 \mathrm{~Hz}), 2.51(\mathrm{ddd}, 1 \mathrm{H}, J=16.4,10.4,2.8 \mathrm{~Hz})$, 2.34-2.11 (m, 2 H), 2.10-2.00 (m, 1 H), 2.00 (s, 3 H), 1.53-1.45 (m, 6 H), 1.35-1.29 (m, 6 $\mathrm{H}), 0.89(\mathrm{t}, 9 \mathrm{H}, J=7.6 \mathrm{~Hz}), 0.86(\mathrm{dd}, 6 \mathrm{H}, J=9.2,7.6 \mathrm{~Hz}) ;{ }^{13} \mathrm{C} \mathrm{NMR}\left(100 \mathrm{MHz}, \mathrm{CDCl}_{3}\right)$ $\delta$ 168.0, 63.4, 38.9, $29.4\left(J_{\mathrm{Sn}}{ }^{117 / 119}=10.6 \mathrm{~Hz}\right), 28.9,27.7\left(J_{\mathrm{Sn}}{ }^{117 / 119}=26.9 \mathrm{~Hz}\right), 19.6,13.9$, $9.3\left(J_{\mathrm{Sn}}{ }^{117 / 119}=151.8 \mathrm{~Hz}\right) ; \mathrm{IR}\left(\mathrm{CHCl}_{3}\right) 1630(\mathrm{w}) \mathrm{cm}^{-1} ; \mathrm{MS}(\mathrm{EI}, 70 \mathrm{eV}) \mathrm{m} / z$ (rel int) 374.2 (2, $\left.\mathrm{M}^{+}\right), 316.2$ (18), 260.2 (29), 204.1 (40), 179.1 (100), 121.0 (37), 85.1 (40), 57.2 (85); HRMS (EI, $70 \mathrm{eV}$ ) calcd for $\mathrm{C}_{13} \mathrm{H}_{26} \mathrm{NSn}\left(\left[\mathrm{M}-\mathrm{C}_{4} \mathrm{H}_{9}\right]^{+}\right)$316.1087; found 316.1102 . 


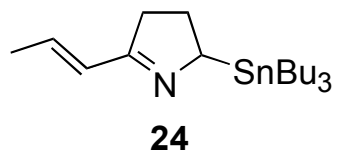

5-(Prop-1-enyl)-2-(tri-n-butylstannyl)-3,4-dihydro-2H-pyrrole (24): To a flask containing stannane $\mathbf{2 b}(60 \mathrm{mg}, 0.15 \mathrm{mmol})$ and [1,3-bis(diphenylphosphinopropane)] dichloronickel (II) (4 mg, $0.0075 \mathrm{mmol})$ in toluene $(1.5 \mathrm{~mL})$ was added allylmagnesium bromide in a dropwise fashion $(0.22 \mathrm{~mL}$ of a $0.817 \mathrm{M}$ solution in diethyl ether, 0.18 mmol). After 15 min., the mixture was diluted with water and extracted with hexanes $(4 \mathrm{x})$. The combined organic phases were dried $\left(\mathrm{Na}_{2} \mathrm{SO}_{4}\right)$, filtered, and concentrated. Chromatography (0-5\% ethyl acetate/hexanes gradient) yielded $18.7 \mathrm{mg}(31 \%)$ of the title compound $\mathbf{2 4}$ as a clear colorless oil. Data for 24: $\mathrm{R}_{f}=0.48$ (20\% ethyl acetate/hexanes); ${ }^{1} \mathrm{H}$ NMR $\left(500 \mathrm{MHz}, \mathrm{CDCl}_{3}\right) \delta 6.40(\mathrm{~d}, 1 \mathrm{H}, J=14.5 \mathrm{~Hz}), 6.06-5.99(\mathrm{~m}$, $1 \mathrm{H}), 4.28(\mathrm{~d}, 1 \mathrm{H}, J=8.5 \mathrm{~Hz}), 2.75(\mathrm{ddd}, 1 \mathrm{H}, J=16.0,9.5,2.5 \mathrm{~Hz}), 2.38-2.30(\mathrm{~m}, 1 \mathrm{H})$, 2.21-2.12 (m, $4 \mathrm{H}), 1.87(\mathrm{~d}, 3 \mathrm{H}, J=6.5 \mathrm{~Hz}), 1.53-1.46(\mathrm{~m}, 6 \mathrm{H}), 1.34-1.27(\mathrm{~m}, 6 \mathrm{H})$, 0.91-0.86 (m, $15 \mathrm{H}) ;{ }^{13} \mathrm{C} \mathrm{NMR}\left(125 \mathrm{MHz}, \mathrm{CDCl}_{3}\right) \delta 167.4,134.0,128.9,64.0,33.7,29.2$, 28.3, 27.4, 18.5, 13.7, 9.4; IR $\left(\mathrm{CHCl}_{3}\right) 1652(\mathrm{w}) \mathrm{cm}^{-1}$; MS (EI, $\left.70 \mathrm{eV}\right), \mathrm{m} / \mathrm{z}$ (rel int) 399.1 (2, $\left.\mathrm{M}^{+}\right), 342.0$ (4), 291.0 (5), 235.0 (16), 150.1 (100), 108.1 (35); HRMS (EI, 70 eV) calcd for $\mathrm{C}_{19} \mathrm{H}_{37} \mathrm{NSn}\left(\mathrm{M}^{+}\right)$399.1948; found 399.1953.

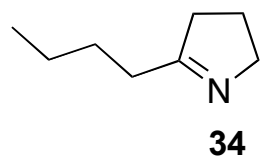

5-n-Butyl-3,4-dihydro-2H-pyrrole (34): Thioimidate $\mathbf{3 3}^{23}$ (23 mg, $0.20 \mathrm{mmol}$ ), [1,3bis(diphenylphosphinopropane)]dichloronickel (II) (5 mg, $0.01 \mathrm{mmol})$ were added to toluene $(2.0 \mathrm{~mL}) \quad n$-Butylmagnesium chloride $(0.11 \mathrm{~mL}$ of a $2.0 \mathrm{M}$ solution in THF, 0.22 mmol) was added in a dropwise fashion. After 30 min., the mixture was diluted with 
water and extracted with pentane $(3 \mathrm{x})$. The combined organic phases were dried $\left(\mathrm{Na}_{2} \mathrm{SO}_{4}\right)$, filtered, and concentrated. Chromatography (0-50\% ethyl acetate/hexanes gradient) provided $19 \mathrm{mg}(75 \%)$ of $\mathbf{3 4}$ as a clear colorless oil. ${ }^{1} \mathrm{H}$ NMR matched that as reported in the literature. ${ }^{10}$

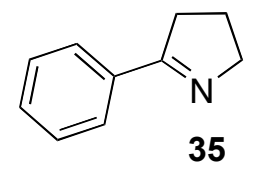

5-Phenyl -3,4-dihydro-2H-pyrrole (35): Thioimidate $\mathbf{3 3}^{23}$ (23 mg, $0.20 \mathrm{mmol}$ ), [1,3bis(diphenylphosphinopropane)]dichloronickel (II) $(5 \mathrm{mg}, 0.01 \mathrm{mmol})$ were added to toluene $(2.0 \mathrm{~mL}) n$-Butylmagnesium chloride $(0.11 \mathrm{~mL}$ of a $2.0 \mathrm{M}$ solution in THF, 0.22 mmol) was added in a dropwise fashion. After 30 min., the mixture was diluted with water and extracted with pentane $(3 \mathrm{x})$. The combined organic phases were dried $\left(\mathrm{Na}_{2} \mathrm{SO}_{4}\right)$, filtered, and concentrated. Kugelrohr distillation $\left(100{ }^{\circ} \mathrm{C} @ 20 \mathrm{~mm} \mathrm{Hg}\right)$ provided $23 \mathrm{mg}(80 \%)$ of $\mathbf{3 5}$ as a clear colorless oil. ${ }^{1} \mathrm{H}$ NMR matched that as reported in the literature. ${ }^{10}$

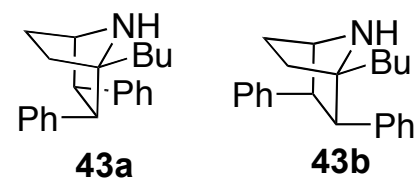

$\left(1 R^{*}, 2 S^{*}, 3 S^{*}, 4 S^{*}\right)-1-B u t y l-2,3-d i p h e n y l-7-a z a b i c y c l o[2.2 .1] h e p t a n e ~(43 a)$ and

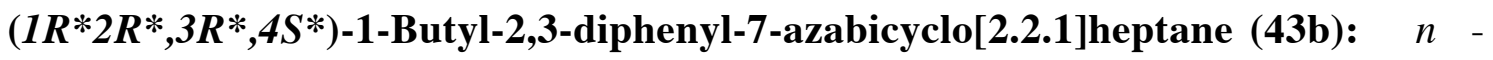
Butyllithium (17 $\mu \mathrm{L}$ of a $2.5 \mathrm{M}$ solution in hexanes, $0.043 \mathrm{mmol}$ ) was added to a solution of stannane 21 (15 mg, $0.036 \mathrm{mmol})$ and trans-stilbene (13 mg, $0.072 \mathrm{mmol})$ in THF $(0.36 \mathrm{~mL})$ cooled to $-78{ }^{\circ} \mathrm{C}$. After $30 \mathrm{~min}$., the reaction was quenched with $0.1 \mathrm{~mL}$ of methanol and warmed to room temperature. The solvent was removed in vacuo and the residue columned directly (10-50\% ethyl acetate:hexanes gradient) to give $5.4 \mathrm{mg}$ of $\mathbf{4 3 a}$ 
and $4.2 \mathrm{mg}$ of $\mathbf{4 3 b}$ (86\% combined yield, 1.3:1) as clear oils. Data for $\mathbf{4 3 a}: \mathbf{R}_{f}=0.11$ (20\% ethyl acetate/hexanes); ${ }^{1} \mathrm{H}$ NMR (500 Mhz, $\left.\mathrm{CDCl}_{3}\right) \delta$ 7.34-7.24 (m, $\left.10 \mathrm{H}\right), 3.78(\mathrm{~d}$, $1 \mathrm{H}, J=5.0 \mathrm{~Hz}), 3.22(\mathrm{~d}, 1 \mathrm{H}, J=6.5 \mathrm{~Hz}), 3.06(\mathrm{dd}, 1 \mathrm{H}, J=6.5,2.0 \mathrm{~Hz}), 1.97(\mathrm{br} \mathrm{s}, 1$ H), 1.92 (dddd, $1 \mathrm{H}, J=12.7,12.5,5.0,5.0 \mathrm{~Hz}), 1.83$ (ddd, $1 \mathrm{H}, J=10.6,9.3,5.0 \mathrm{~Hz}$ ), 1.72-1.64 (m, $2 \mathrm{H}), 1.56-1.47(\mathrm{~m}, 2 \mathrm{H}), 1.39-1.28(\mathrm{~m}, 4 \mathrm{H}), 0.90(\mathrm{t}, 3 \mathrm{H}, J=7.0 \mathrm{~Hz}) ;{ }^{13} \mathrm{C}$ NMR (100 Mhz, $\left.\mathrm{CDCl}_{3}\right) \delta 129.1,128.7,128.4,126.9,126.7,71.2,63.1,62.7,55.4,33.1$, 33.1, 27.7, 27.2, 23.6, 14.3; IR (neat) 3393 (br, m), 1600 (w), 1495 (w), 1449 (w) cm MS (EI, $70 \mathrm{eV}$ ) m/z (rel int) $305.3\left(5, \mathrm{M}^{+}\right), 205.2$ (38), 125.1 (100), 82.1 (54); HRMS (EI, $70 \mathrm{eV}$ ) calcd for $\mathrm{C}_{22} \mathrm{H}_{27} \mathrm{~N}\left(\mathrm{M}^{+}\right)$305.2143; found 305.2147. Data for 43b: $\mathrm{R}_{f}=0.06(20 \%$ ethyl acetate/hexanes); ${ }^{1} \mathrm{H}$ NMR (500 Mhz, $\left.\mathrm{CDCl}_{3}\right) \delta 7.41(\mathrm{~d}, 2 \mathrm{H}, J=7.0 \mathrm{~Hz}), 7.30-7.20$ (m, $8 \mathrm{H}), 3.89(\mathrm{dd}, 1 \mathrm{H}, J=4.5,4.0 \mathrm{~Hz}), 3.61(\mathrm{dd}, 1 \mathrm{H}, J=5.5,5.5 \mathrm{~Hz}), 2.98(\mathrm{~d}, 1 \mathrm{H}, J=$ $7.0 \mathrm{~Hz}$ ), 2.12 (br s, $1 \mathrm{H}), 1.70-1.50$ (m, $4 \mathrm{H}), 1.41-1.38$ (m, $1 \mathrm{H}), 1.30$ (ddd, $1 \mathrm{H}, J=12.5$, 11.3, $6.0 \mathrm{~Hz}$ ), 1.24-1.15 (m, $2 \mathrm{H}), 1.10$ (hex, $1 \mathrm{H}, J=7.5 \mathrm{~Hz}$ ), 0.99 (hex, $1 \mathrm{H}, J=7.5 \mathrm{~Hz}$ ), $0.67(\mathrm{t}, 3 \mathrm{H}, J=7.5 \mathrm{~Hz}) ;{ }^{13} \mathrm{C}$ NMR $\left(100 \mathrm{Mhz}, \mathrm{CDCl}_{3}\right) \delta 129.2,128.4,128.3,128.1$, $126.9,126.4,126.3,72.0,61.0,59.5,55.5,36.9,32.9,27.1,26.2,23.4,13.9 ;$ IR (neat) 3400 (br, m), 1600 (w), 1495 (w), 1454(w) $\mathrm{cm}^{-1}$; MS (EI, $70 \mathrm{eV}$ ) m/z (rel int) 305.3 (4, $\mathrm{M}^{+}$), 205.2 (25), 125.1 (100), 82.1 (44), 49.0 (46); HRMS (EI, $70 \mathrm{eV}$ ) calcd for $\mathrm{C}_{22} \mathrm{H}_{27} \mathrm{~N}$ $\left(\mathrm{M}^{+}\right)$305.2143; found 305.2141.

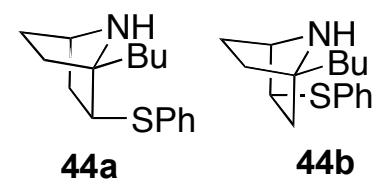

$\left(1 R^{*}, 3 R^{*}, 4 S^{*}\right)$-1-Butyl-3-phenylsulfanyl-7-azabicyclo[2.2.1]heptane (44a) and $\left(1 R^{*}, 2 S^{*}, 4 S^{*}\right)$-1-Butyl-2-phenylsulfanyl-7-azabicyclo[2.2.1]heptane $(44 \mathrm{~b}): n$ Butyllithium ( $17 \mu \mathrm{L}$ of a $2.5 \mathrm{M}$ solution in hexanes, $0.043 \mathrm{mmol})$ was added in a 
dropwise fashion to a solution of stannane $21(15 \mathrm{mg}, 0.036 \mathrm{mmol})$ and phenyl vinyl sulfide $(9.4 \mu \mathrm{L}, 9.8 \mathrm{mg}, 0.072 \mathrm{mmol})$ in THF $(0.36 \mathrm{~mL})$ cooled to $-78^{\circ} \mathrm{C}$. After $30 \mathrm{~min}$., $0.1 \mathrm{~mL}$ of methanol was added and the mixture was warmed to room temperature. The solvent was removed in vacuo and the residue was subjected to column chromatography directly (10-50\% ethyl acetate/hexanes gradient) to yield $2.3 \mathrm{mg}$ of $\mathbf{4 4 a}$ and $1.7 \mathrm{mg}$ of 44b (43\% combined yield, 1.3:1) both as colorless oils. Data for 44a: $\mathbf{R}_{f}=0.04(20 \%$ ethyl acetate/hexanes); ${ }^{1} \mathrm{H}$ NMR (500 MHz, $\mathrm{CDCl}_{3}$ ) $\delta$ 7.35-7.27 (m, $4 \mathrm{H}$ ), 7.21-7.18 (m, 1 H), $3.48(\mathrm{dd}, 1 \mathrm{H}, J=7.8,3.5 \mathrm{~Hz}), 3.39(\mathrm{~d}, 1 \mathrm{H}, J=5.0 \mathrm{~Hz}), 2.06(\mathrm{dd}, 2 \mathrm{H}, J=13.0,8.0$ Hz), 1.85-1.83 (m, $1 \mathrm{H}), 1.74-1.70(\mathrm{~m}, 2 \mathrm{H}), 1.37-1.33$ (m, $6 \mathrm{H}), 1.15$ (dt, $2 \mathrm{H}, J=13.0$, $4.0 \mathrm{~Hz}), 0.92(\mathrm{t}, 3 \mathrm{H}, J=7.0 \mathrm{~Hz}) ;{ }^{13} \mathrm{C} \mathrm{NMR}\left(100 \mathrm{MHz}, \mathrm{CDCl}_{3}\right) \delta$ 129.9, 129.2, 126.4, 125.7, 61.3, 43.0, 35.2, 33.6, 30.5, 29.9, 29.7, 28.1, 23.6, 14.3; IR (neat) 3360 (br, w), $3080(\mathrm{w}), 1580(\mathrm{w}) \mathrm{cm}^{-1}$; MS (EI, $\left.70 \mathrm{eV}\right) \mathrm{m} / \mathrm{z}$ (rel int) $261.2\left(18, \mathrm{M}^{+}\right), 205.2(100), 125.1$ (79), 82.1 (56), 57.1 (34); HRMS (EI, $70 \mathrm{eV}$ ) calcd for $\mathrm{C}_{16} \mathrm{H}_{23} \mathrm{NS}\left(\mathrm{M}^{+}\right)$261.1551; found 261.1540. Data for 44b: $\mathrm{R}_{f}=0.14$ (20\% ethyl acetate/hexanes); ${ }^{1} \mathrm{H}$ NMR $(500 \mathrm{MHz}$, $\left.\mathrm{CDCl}_{3}\right) \delta 7.36-7.33(\mathrm{~m}, 2 \mathrm{H}), 7.28-7.25(\mathrm{~m}, 2 \mathrm{H}), 7.18-7.14(\mathrm{~m}, 1 \mathrm{H}), 4.05(\mathrm{t}, 1 \mathrm{H}, J=7.5$ Hz), 3.11 (ddd, $1 \mathrm{H}, J=13.0,9.5,6.0 \mathrm{~Hz}), 3.04(\mathrm{ddd}, 1 \mathrm{H}, J=12.6,9.8,5.5 \mathrm{~Hz}), 2.46-$ $2.41(\mathrm{~m}, 1 \mathrm{H}), 2.32(\mathrm{t}, 1 \mathrm{H}, J=8.0 \mathrm{~Hz}), 2.06$ (dddd, $1 \mathrm{H}, J=12.7,9.5,8.0,5.0 \mathrm{~Hz}), 1.99$ 1.91 (m, $1 \mathrm{H}), 1.81-1.75(\mathrm{~m}, 1 \mathrm{H}), 1.59-1.52(\mathrm{~m}, 4 \mathrm{H}), 1.33$ (hex, $2 \mathrm{H}, J=7.5 \mathrm{~Hz}), 0.92$ (t, $3 \mathrm{H}, J=7.0 \mathrm{~Hz}) ;{ }^{13} \mathrm{C} \mathrm{NMR}\left(100 \mathrm{MHz}, \mathrm{CDCl}_{3}\right) \delta 129.1,129.0,125.9,71.7,37.2,36.5$, 33.9, 31.1, 30.5, 29.9, 29.0, 22.8, 14.1; IR (neat) 3206 (br, w), $1641(\mathrm{~m}), 1583(\mathrm{~m}) \mathrm{cm}^{-1-}$; MS (EI, $70 \mathrm{eV}) \mathrm{m} / \mathrm{z}$ (rel int) $261.2\left(11, \mathrm{M}^{+}\right), 205.1$ (100), 125.1 (56), 82.1 (55), 57.1 (31); HRMS (EI, $70 \mathrm{eV}$ ) calcd for $\mathrm{C}_{16} \mathrm{H}_{23} \mathrm{NS}\left(\mathrm{M}^{+}\right)$261.1551; found 261.1542 . 


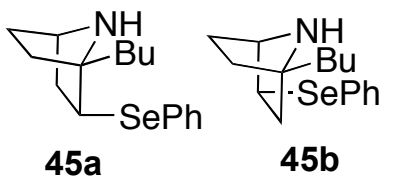

$\left(1 R^{*}, 2 S^{*}, 4 S^{*}\right)$-1-Butyl-2-phenylselanyl-7-azabicyclo[2.2.1]heptane (45a) and $\left(1 R^{*}\right.$, $\left.3 R^{*}, 4 S^{*}\right)$-1-Butyl-3-phenylselanyl-7-azabicyclo[2.2.1]heptane (45b): $n$-Butyllithium (48.0 $\mu \mathrm{L}$ of a $2.5 \mathrm{M}$ solution in hexanes, $0.12 \mathrm{mmol}$ ) was added in a dropwise fashion to a solution of stannane $21(25.0 \mathrm{mg}, 0.060 \mathrm{mmol})$ and phenyl vinyl selenide $(22.0 \mathrm{mg}$, $0.12 \mathrm{mmol})$ in THF $(0.60 \mathrm{~mL})$ at $-78{ }^{\circ} \mathrm{C}$. After $30 \mathrm{~min} ., 0.1 \mathrm{~mL}$ of methanol was added and the solution warmed to room temperature. Solvent was removed in vacuo and the residue columned (1-2\% methanol/methylene chloride gradient) to give $7.3 \mathrm{mg}$ of $\mathbf{4 5 a}$ and $6.5 \mathrm{mg}$ of $\mathbf{4 5 b}$ as oils (74\% combined yield, 1.1:1). Data for $\mathbf{4 5 a}: \mathbf{R}_{f}=0.25(5 \%$ methanol/methylene chloride); ${ }^{1} \mathrm{H}$ NMR (500 MHz, $\left.\mathrm{CDCl}_{3}\right) \delta$ 7.50-7.49 (m, $\left.2 \mathrm{H}\right)$, 7.26$7.21(\mathrm{~m}, 3 \mathrm{H}), 4.02(\mathrm{t}, 1 \mathrm{H}, J=7.5 \mathrm{~Hz}), 3.06(\mathrm{ddd}, 1 \mathrm{H}, J=12.0,9.5,6.0 \mathrm{~Hz}), 3.01$ (ddd, $1 \mathrm{H}, J=12.0,9.5,6.0 \mathrm{~Hz}), 2.50(\mathrm{dqd}, 1 \mathrm{H}, J=17.0,5.0,2.0 \mathrm{~Hz}), 2.41(\mathrm{dt}, 1 \mathrm{H}, J=17.0$, $9.5 \mathrm{~Hz}), 2.31(\mathrm{t}, 2 \mathrm{H}, J=9.0 \mathrm{~Hz}), 2.07-1.98(\mathrm{~m}, 2 \mathrm{H}), 1.86-1.79(\mathrm{~m}, 1 \mathrm{H}), 1.54$ (quint, $2 \mathrm{H}$, $J=7.0 \mathrm{~Hz}), 1.42-1.38(\mathrm{~m}, 1 \mathrm{H}), 1.34(\mathrm{hex}, 2 \mathrm{H}, J=7.0 \mathrm{~Hz}), 0.91(\mathrm{t}, 3 \mathrm{H}, J-8.0 \mathrm{~Hz}) ;{ }^{13} \mathrm{C}$ NMR (100 MHz, $\left.\mathrm{CDCl}_{3}\right) \delta 132.6,129.2,126.8,72.6,37.5,37.4,33.9,28.9,28.7,25.1$, 22.8, 14.1; IR (neat) 3266 (w), 1640 (s), 1578 (s), 1477 (s), 1436 (s) cm ${ }^{-1}$; MS (EI, $70 \mathrm{eV}$ ) $m / z$ (rel int) $309.2\left(24, \mathrm{M}^{+}\right), 232.1$ (20), 138.2 (49), 125.1 (98), 82.1 (100); HRMS (EI, 70 eV) calcd for $\mathrm{C}_{16} \mathrm{H}_{23} \mathrm{Nse}\left(\mathrm{M}^{+}\right)$309.0996; found 309.0997. Data for $\mathbf{4 5 b}: \mathrm{R}_{f}=0.13$ (5\% methanol/methylene chloride); ${ }^{1} \mathrm{H}$ NMR (500 MHz, $\left.\mathrm{CDCl}_{3}\right) \delta 7.51$ (dd, $2 \mathrm{H}, J=7.3,2.5$ Hz), 7.29-7.26 (m, $3 \mathrm{H}), 3.56(\mathrm{dd}, 1 \mathrm{H}, J=8.0,4.0 \mathrm{~Hz}), 3.44(\mathrm{~d}, 1 \mathrm{H}, J=5.0 \mathrm{~Hz}), 2.07$ (dd, $1 \mathrm{H}, J=13.5,8.0 \mathrm{~Hz}$ ), 1.88-1.84 (m, $1 \mathrm{H}), 1.72$ (quint, $2 \mathrm{H}, J=5.0 \mathrm{~Hz}$ ), 1.46-1.34 $(\mathrm{m}, 7 \mathrm{H}), 1.25(\mathrm{t}, 1 \mathrm{H}, J=3.5 \mathrm{~Hz}), 1.22(\mathrm{t}, 1 \mathrm{H}, J=3.5 \mathrm{~Hz}), 0.93(\mathrm{t}, 3 \mathrm{H}, J=6.5 \mathrm{~Hz}) ;{ }^{13} \mathrm{C}$ 
NMR (125 MHz, $\left.\mathrm{CDCl}_{3}\right) \delta 133.4,131.1,129.3,127.3,67.7,61.9,47.4,43.2,35.2,33.6$, 30.4, 28.1, 23.6, 14.3; IR (neat) 3239 (w), 1578 (m), 1476 (s), 1436 (s) cm ${ }^{-1}$; MS (EI, 70 eV) $\mathrm{m} / \mathrm{z}$ (rel int) $309.2\left(3, \mathrm{M}^{+}\right), 152.2$ (12), 125.2 (13), 84.0 (100), 49.0 (99); HRMS (EI, $70 \mathrm{eV}$ ) calcd for $\mathrm{C}_{16} \mathrm{H}_{23} \mathrm{Nse}\left(\mathrm{M}^{+}\right)$309.0996, found 309.1001.

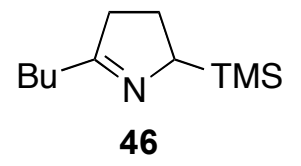

5-Butyl-2-(trimethylsilyl)-3,4-dihydro-2H-pyrrole (46): $n$-Butyllithium was added in a dropwise fashion to a solution of stannane $21(25.0 \mathrm{mg}, 0.060 \mathrm{mmol})$ and trimethylsilyl phenyl acetylene $(21.0 \mathrm{mg}, 0.12 \mathrm{mmol})$ in THF $(0.60 \mathrm{~mL})$ at $-78{ }^{\circ} \mathrm{C}$. After $30 \mathrm{~min}$., the mixture was quenched with $0.1 \mathrm{~mL}$ of methanol and warmed to room temperature. The solvent was removed in vacuo and the residue columned (0-5\% methanol/methylene chloride gradient) to give $6.5 \mathrm{mg}(55 \%)$ of $\mathbf{4 6}$ as an oil. Data for $\mathbf{4 6}: \mathrm{R}_{f}=0.36(5 \%$ methanol/methylene chloride); ${ }^{1} \mathrm{H}$ NMR (500 MHz, $\left.\mathrm{CDCl}_{3}\right) \delta 3.57-3.54(\mathrm{~m}, 1 \mathrm{H}), 2.50$ (dd, $1 \mathrm{H}, J=11.3,4.5 \mathrm{~Hz}), 2.46(\mathrm{dd}, 1 \mathrm{H}, J=11.3,5.5 \mathrm{~Hz}), 2.42-2.28(\mathrm{~m}, 2 \mathrm{H}), 2.05-1.96$ (m, $1 \mathrm{H}), 1.80(\mathrm{ddd}, 1 \mathrm{H}, J=12.9,10.0,5.0 \mathrm{~Hz}), 1.57-1.47(\mathrm{~m}, 2 \mathrm{H}), 1.35$ (hex, $2 \mathrm{H}, J=$ $7.5 \mathrm{~Hz}), 0.91(\mathrm{t}, 3 \mathrm{H}, J=7.5 \mathrm{~Hz}), 0.01(\mathrm{~s}, 9 \mathrm{H}) ;{ }^{13} \mathrm{C} \mathrm{NMR}\left(125 \mathrm{MHz}, \mathrm{CDCl}_{3}\right) \delta 168.6$, 64.6, 37.6, 33.7, 29.4, 24.6, 22.9, 14.1, -3.3; IR (neat) 1634 (w) $\mathrm{cm}^{-1}$; MS (EI, $70 \mathrm{eV}$ ) $\mathrm{m} / \mathrm{z}$ (rel int) $197.2\left(6, \mathrm{M}^{+}\right), 182.2(8), 154.1(64), 73.1$ (100); HRMS (EI, $70 \mathrm{eV}$ ) calcd for $\mathrm{C}_{11} \mathrm{H}_{23} \mathrm{Nsi}\left(\mathrm{M}^{+}\right)$197.1600; found 197.1597.

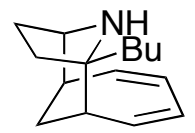

$47 a$

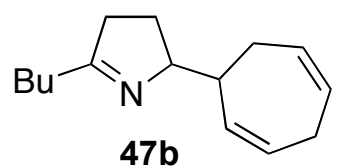

47b

$\left(1 R^{*}, 2 R^{*}, 5 S^{*}, 6 S^{*}\right)$-2-Butyl-12-azatricyclo[4.4.1.1 $\left.{ }^{2,5}\right]$ dodeca-7,9-diene $(47 \mathrm{a})$ and 5Butyl-2-cyclohepta-2',5'-dienyl-3,4-dihydro-2H-pyrrole (47b): $n$-Butyllithium (17 $\mu \mathrm{L}$ 
of a $2.5 \mathrm{M}$ solution in hexanes, $0.043 \mathrm{mmol}$ ) was added in a dropwise fashion to a solution of stannane 21 (15 mg, $0.036 \mathrm{mmol})$ and cycloheptatriene (11 $\mu \mathrm{L}, 9.9 \mathrm{mg}, 0.11$ $\mathrm{mmol})$ in THF $(0.36 \mathrm{~mL})$ cooled to $-78{ }^{\circ} \mathrm{C}$. After 30 , min $0.1 \mathrm{~mL}$ of methanol was added and the solution was warmed to room temperature. The solvent was removed in vacuo and the residue was columned directly to yield $2.9 \mathrm{mg}$ of $47 \mathbf{a}$ and $2.3 \mathrm{mg}$ of $\mathbf{4 7 \mathbf { b }}$ (67\% combined yield, $1.3: 1)$ both as clear oils. Data for $\mathbf{4 7 a}: \mathbf{R}_{f}=0.06$ (20\% ethyl acetate/hexanes); ${ }^{1} \mathrm{H}$ NMR $\left(500 \mathrm{MHz}, \mathrm{CDCl}_{3}\right) \delta 6.01-5.96(\mathrm{~m}, 2 \mathrm{H})$, 5.89-5.84 (m, $\left.2 \mathrm{H}\right)$, $3.38(\mathrm{~d}, 1 \mathrm{H}, J=7.0 \mathrm{~Hz}), 2.46-2.44(\mathrm{~m}, 1 \mathrm{H}), 2.38-2.33(\mathrm{~m}, 2 \mathrm{H}), 2.10$ (tdd, $1 \mathrm{H}, J=12.3$, 7.0, $6.5 \mathrm{~Hz}), 1.89$ (dd, $1 \mathrm{H}, J=9.3,4.0 \mathrm{~Hz}), 1.86(\mathrm{dd}, 1 \mathrm{H}, J=9.3,5.0 \mathrm{~Hz}), 1.74$ (ddd, 1 $\mathrm{H}, J=12.6,9.3,4.5 \mathrm{~Hz}), 1.68(\mathrm{ddd}, 1 \mathrm{H}, J=12.3,12.3,4.5 \mathrm{~Hz}), 1.58-1.49(\mathrm{~m}, 2 \mathrm{H})$, $1.41-1.30(\mathrm{~m}, 4 \mathrm{H}), 0.90(\mathrm{t}, 3 \mathrm{H}, J=7.0 \mathrm{~Hz}) ;{ }^{13} \mathrm{C} \mathrm{NMR}\left(100 \mathrm{MHz}, \mathrm{CDCl}_{3}\right) \delta 136.7,135.6$, $126.8,68.8,61.2,43.2,42.4,39.8,35.1,30.5,29.5,27.4,23.7,23.4,14.3$; IR (neat) 3312 (br, w), $1456(\mathrm{~m}) \mathrm{cm}^{-1}$; MS (EI, $\left.70 \mathrm{eV}\right) \mathrm{m} / z$ (rel int) $217.2\left(44, \mathrm{M}^{+}\right), 125.1$ (89), 82.1 (100); HRMS (EI, $70 \mathrm{eV}$ ) calcd for $\mathrm{C}_{15} \mathrm{H}_{23} \mathrm{~N}\left(\mathrm{M}^{+}\right)$217.1830; found 217.1831. Data for 47b: $\mathrm{R}_{f}=0.24\left(20 \%\right.$ ethyl acetate/hexanes); ${ }^{1} \mathrm{H}$ NMR $\left(500 \mathrm{MHz}, \mathrm{CDCl}_{3}\right) \delta 5.75-5.62(\mathrm{~m}$, $4 \mathrm{H}), 3.98(\mathrm{~d}, 1 \mathrm{H}, J=6.5 \mathrm{~Hz}), 2.97-2.91(\mathrm{~m}, 1 \mathrm{H}), 2.80-2.75(\mathrm{~m}, 2 \mathrm{H}), 2.53-2.32(\mathrm{~m}, 4$ H), 2.24-2.17 (m, $1 \mathrm{H}), 2.13-2.09$ (m, $1 \mathrm{H}), 1.96$ (dddd, $1 \mathrm{H}, J=12.8,9.8,7.8,4.5 \mathrm{~Hz}$ ), 1.58-1.50 (m, $3 \mathrm{H}), 1.35$ (hex, $2 \mathrm{H}, J=7.5 \mathrm{~Hz}), 0.92(\mathrm{t}, 3 \mathrm{H}, J=7.5 \mathrm{~Hz}) ;{ }^{13} \mathrm{C}$ NMR (100 $\left.\mathrm{MHz}, \mathrm{CDCl}_{3}\right) \delta 159.7,134.34,129.9,128.3,42.6,37.4,34.0,30.5,29.2,29.1,28.6,26.0$, 22.9, 14.1; IR (neat) $1642(\mathrm{~m}), 1460(\mathrm{~m}) \mathrm{cm}^{-1}$; MS (EI, $\left.70 \mathrm{eV}\right) \mathrm{m} / z$ (rel int) 217.3 (27, $\mathrm{M}^{+}$), 188.2 (98), 175.2 (48), 124.2 (95), 82.1 (100); HRMS (EI, $70 \mathrm{eV}$ ) calcd for $\mathrm{C}_{15} \mathrm{H}_{23} \mathrm{~N}$ $\left(\mathrm{M}^{+}\right)$217.1830; found 217.1828. 


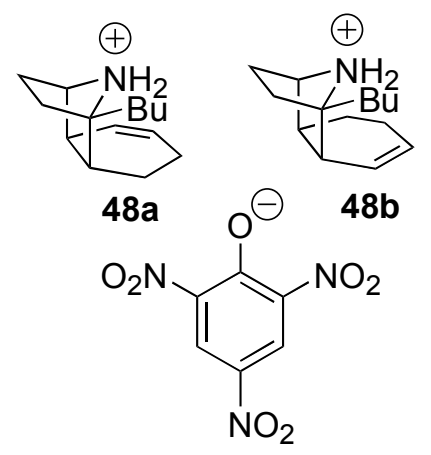

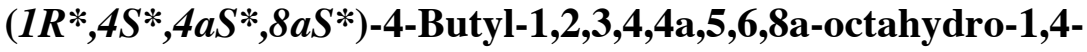

epiazanonaphthalenium picrate (48a) and $\left(1 R^{*}, 4 S^{*}, 4 a R^{*}, 8 a R^{*}\right)-1$-Butyl1,2,3,4,4a,5,6,8a-octahydro-1,4-epiazanonaphthalenium picrate $(48 \mathrm{~b}): n$ Butyllithium ( $48 \mu \mathrm{L}$ of a $2.5 \mathrm{M}$ solution in hexanes, $0.12 \mathrm{mmol}$ ) was added in a dropwise fashion to a solution of stannane $21(25 \mathrm{mg}, 0.060 \mathrm{mmol})$ and 1,3-cyclohexadiene (17 $\mu \mathrm{L}, 14 \mathrm{mg}, 0.18 \mathrm{mmol})$ in THF $(0.60 \mathrm{~mL})$ cooled to $-78{ }^{\circ} \mathrm{C}$. After $30 \mathrm{~min} ., 0.1 \mathrm{~mL}$ of methanol was added and the solution warmed to room temperature. Picric acid $(0.3 \mathrm{~mL}$ of a $0.2 \mathrm{M}$ solution in ethanol, $0.060 \mathrm{mmol}$ ) was added in a dropwise fashion. The mixture was then concentrated and the residue was columned (0-1\% methanol/methylene chloride) to give $18 \mathrm{mg}$ ( $68 \%$ combined yield) of the picrate salts $48 \mathrm{a}$ and $\mathbf{4 8 b}(1.5: 1$ by ${ }^{1} \mathrm{H}$ NMR) as a yellow oil. Data for 48a/b: $\mathrm{R}_{f}=0.4\left(10 \% \mathrm{MeOH} / \mathrm{CH}_{2} \mathrm{Cl}_{2}\right) ;{ }^{1} \mathrm{H} \mathrm{NMR}(500$ $\left.\mathrm{MHz}, \mathrm{CDCl}_{3}\right) \delta 8.86(\mathrm{~s}, 2 \mathrm{H}), 6.18(\mathrm{dddd}, 0.60 \mathrm{H}, J=10.3,4.4,4.3,2.0 \mathrm{~Hz}), 5.99-5.95$ (m, 0.40 H), 5.71 (ddd, 0.60 H, $J=10.3,1.8,1.5 \mathrm{~Hz}), 5.63-5.61(\mathrm{~m}, 0.40 \mathrm{H}), 3.93(\mathrm{~d}, 0.4$ $\mathrm{H}, J=4.5 \mathrm{~Hz}), 3.91(\mathrm{~d}, 0.60 \mathrm{H}, J=4.0 \mathrm{~Hz}), 2.62-2.57(\mathrm{~m}, 1 \mathrm{H}), 2.42-2.21(\mathrm{~m}, 2 \mathrm{H}), 2.16-$ $1.71(\mathrm{~m}, 9 \mathrm{H}), 1.53-1.29(\mathrm{~m}, 6 \mathrm{H}), 0.94(\mathrm{t}, 1.8 \mathrm{H}, J=7.0 \mathrm{~Hz}), 0.93(\mathrm{t}, 1.2 \mathrm{H}, J=7.5 \mathrm{~Hz})$; ${ }^{13} \mathrm{C}$ NMR $\left(100 \mathrm{MHz}, \mathrm{CDCl}_{3}\right) \delta 161.9,141.9,133.8,132.5,128.7,126.7,126.4,124.4$, $75.9,75.2,64.5,64.0,42.0,41.5,40.3,32.2,31.5,30.0,29.3,28.7,27.6,27.4,27.2,25.1$, 23.3, 23.2, 22.3, 21.9, 21.4, 14.0; IR (neat) 3213 (w), 1628 (s), 1607 (s), 1566 (s), 1538 
(s), $1316(\mathrm{~s}) \mathrm{cm}^{-1}$; MS (EI, $\left.70 \mathrm{eV}\right) \mathrm{m} / z$ (rel int) $205.2\left(17,[\mathrm{M}-\mathrm{H}]^{+}\right), 125.1$ (91), 82.1

(100), 68.1 (34), 55.1 (18); HRMS (ES, positive cation) calcd for $\mathrm{C}_{14} \mathrm{H}_{24} \mathrm{~N}\left([\mathrm{M}+\mathrm{H}]^{+}\right)$ 206.1909; found 206.1910.

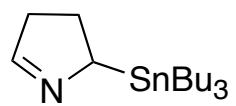

49

5-(Tri-n-butylstannyl)-2-pyrroline (49): Stannane 56 (346 mg, $0.70 \mathrm{mmol})$ and hydrazine monohydrate $(1.80 \mathrm{~mL}, 37.1 \mathrm{mmol})$ were dissolved in ethanol $(15 \mathrm{~mL})$ and heated under reflux. After $5 \mathrm{~h}$, the reaction was concentrated, diluted with ether, washed with water $(3 \mathrm{x})$, dried $\left(\mathrm{Na}_{2} \mathrm{SO}_{4}\right)$, filtered, and concentrate to provide an oil. Chromatography (0-5\% ethyl acetate/hexanes gradient) afforded $193 \mathrm{mg}$ (78\%) of 49 as an oil. Data for 49: $\mathrm{R}_{f}=0.28$ (10\% ethyl acetate/hexanes); ${ }^{1} \mathrm{H}$ NMR $\left(360 \mathrm{MHz}, \mathrm{CDCl}_{3}\right) \delta$ $7.32\left(\mathrm{~s}, 1 \mathrm{H},{ }^{4} J\left({ }^{117 / 119} \mathrm{Sn}-{ }^{1} \mathrm{H}\right)=16.8 \mathrm{~Hz}\right), 4.22-4.04(\mathrm{~m}, 1 \mathrm{H}), 2.63-2.55(\mathrm{~m}, 1 \mathrm{H}), 2.37-2.26$ (m, $1 \mathrm{H}), 2.10-1.93(\mathrm{~m}, 2 \mathrm{H}), 1.58-1.41(\mathrm{~m}, 6 \mathrm{H}), 1.28$ (hex, $6 \mathrm{H}, J=7.4 \mathrm{~Hz}), 0.98-0.68$ $(\mathrm{m}, 15 \mathrm{H}) ;{ }^{13} \mathrm{C}$ NMR $\left(90 \mathrm{MHz}, \mathrm{CDCl}_{3}\right) \delta 160.0,63.5,36.3,29.1,27.4,26.4,13.6,9.0$; IR (neat) $1708(\mathrm{w}), 1596(\mathrm{~m}) ; \mathrm{MS}\left(\mathrm{CI}, \mathrm{NH}_{3}\right) \mathrm{m} / \mathrm{z}$ (rel int) 360 (100, M+H); HRMS (CI, $\mathrm{NH}_{3}$ ) calcd for $\mathrm{C}_{16} \mathrm{H}_{34} \mathrm{NSn}[\mathrm{M}+\mathrm{H}]^{+} 360.1713$; found 360.1701 .

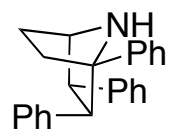

50

$\left(1 R^{*}, 2 S^{*}, 3 S^{*}, 4 S^{*}\right)-1,2,3$-Triphenyl-7-azabicyclo[2.2.1]heptane (50): $n$-Butyllithium $(118 \mu \mathrm{L}$ of a $1.7 \mathrm{M}$ solution in hexanes, $0.20 \mathrm{mmol}$ ) was added in a dropwise fashion to a solution of stannane $19(29.0 \mathrm{mg}, 0.067 \mathrm{mmol})$ and trans-stilbene $(24.1 \mathrm{mg}, 0.13 \mathrm{mmol})$ in THF $(0.70 \mathrm{~mL})$ at $-78{ }^{\circ} \mathrm{C}$. After $30 \mathrm{~min}$., the reaction was quenched with $0.1 \mathrm{~mL}$ methanol and warmed to $25{ }^{\circ} \mathrm{C}$. The solvent was removed in vacuo and the organic 
residue was purified by chromatography ( $0-10 \%$ ethyl acetate/hexanes gradient) to give $9.2 \mathrm{mg}(40 \%)$ of $\mathbf{5 0}$ as an oil. Data for $\mathbf{5 0}: \mathbf{R}_{f}=0.32$ (20\% ethyl acetate/hexanes); ${ }^{1} \mathrm{H}$ NMR (500 MHz, $\left.\mathrm{CDCl}_{3}\right) \delta 7.50(\mathrm{~d}, 2 \mathrm{H}, J=7.0 \mathrm{~Hz}), 7.29-7.18(\mathrm{~m}, 11 \mathrm{H}), 6.89(\mathrm{dd}, 2 \mathrm{H}$, $J=8.0,2.8 \mathrm{~Hz}), 3.90(\mathrm{~d}, 1 \mathrm{H}, J=5.0 \mathrm{~Hz}), 3.26-3.23(\mathrm{~m}, 2 \mathrm{H}), 2.31$ (ddd, $1 \mathrm{H}, J=12.7$, 8.5, $5.0 \mathrm{~Hz}), 2.06-1.93(\mathrm{~m}, 2 \mathrm{H}), 1.52(\mathrm{tdd}, 1 \mathrm{H}, J=12.5,5.0,2.0 \mathrm{~Hz}) ;{ }^{13} \mathrm{C}$ NMR $(100$ $\left.\mathrm{MHz}, \mathrm{CDCl}_{3}\right) \delta 146.5,142.4,138.5,129.3,128.6,128.1,127.9,127.4,127.3,127.0$, 126.8, 126.3, 74.1, 66.8, 63.2, 54.2, 34.0, 29.5; IR (neat) 1599 (m), 1495 (m), 1447 (m) $\mathrm{cm}^{-1}$; MS (EI, $\left.70 \mathrm{eV}\right) \mathrm{m} / z$ (rel int) $325.2\left(1, \mathrm{M}^{+}\right), 202.2$ (13), 145.1 (100), 104.1 (12), 77.1 (7); HRMS (ES, pos. ion) calcd for $\mathrm{C}_{24} \mathrm{H}_{24} \mathrm{~N}[\mathrm{M}+\mathrm{H}]^{+}$326.1909; found 326.1906 .

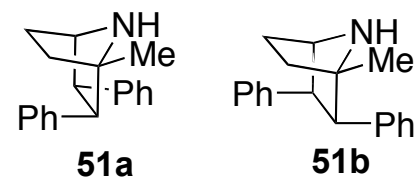

$\left(1 R^{*}, 2 S^{*}, 3 S^{*}, 4 R^{*}\right)$-1-Methyl-2,3-diphenyl-7-azabicyclo[2.2.1]heptane (51a) and $\left(1 R^{*}, 2 R^{*}, 3 R^{*}, 4 S^{*}\right)-1-M e t h y l-2,3-d i p h e n y l-7-a z a b i c y c l o[2.2 .1]$ heptane (51b): $\quad n-$ Butyllithium (50 $\mu \mathrm{L}$ of a $2.5 \mathrm{M}$ solution in hexanes, $0.12 \mathrm{mmol}$ ) was added to a solution of stannane $22(25 \mathrm{mg}, 0.062 \mathrm{mmol})$ and trans-stilbene $(22 \mathrm{mg}, 0.12 \mathrm{mmol})$ in THF (0.62 $\mathrm{mL}$ ) cooled to $-78{ }^{\circ} \mathrm{C}$. After $30 \mathrm{~min}$., the reaction was quenched with $0.1 \mathrm{~mL}$ of methanol and warmed to room temperature. The solvent was removed in vacuo and the residue columned directly (2-5\% methanol/methylene chloride gradient) to give $15 \mathrm{mg}$ of 51a and 51b (89\% combined yield, 1.5:1) as a clear oil. Data for 51a/b: $\mathbf{R}_{f}=0.36(10 \%$ methanol/methylene chloride); ${ }^{1} \mathrm{H}$ NMR (500 MHz, $\left.\mathrm{CDCl}_{3}\right) \delta$ 7.40-7.14 (m, $\left.10 \mathrm{H}\right), 3.91$ $(\mathrm{t}, 0.41 \mathrm{H}, J=4.5 \mathrm{~Hz}), 3.80(\mathrm{~d}, 0.59 \mathrm{H}, J=5.0 \mathrm{~Hz}), 3.68(\mathrm{t}, 0.41 \mathrm{H}, J=5.5 \mathrm{~Hz}), 3.25(\mathrm{~d}$, $0.59 \mathrm{H}, J=6.5 \mathrm{~Hz}), 2.97(\mathrm{dd}, 0.59 \mathrm{H}, J=6.5,2.5 \mathrm{~Hz}), 2.93(\mathrm{~d}, 0.41 \mathrm{H}, J=6.5 \mathrm{~Hz}), 2.02-$ $1.60(\mathrm{~m}, 2 \mathrm{H}), 1.51(\mathrm{td}, 1 \mathrm{H}, J=11.8,7.0 \mathrm{~Hz}), 1.32(\mathrm{~s}, 1.76 \mathrm{H}), 1.17-1.12(\mathrm{~m}, 1 \mathrm{H}), 0.98$ 
(s, $1.24 \mathrm{H}) ;{ }^{13} \mathrm{C}$ NMR $\left(100 \mathrm{MHz}, \mathrm{CDCl}_{3}\right) \delta 146.2,141.3,139.7,129.0,128.8,128.7$, $128.5,128.4,128.1,126.9,126.8,126.5,126.3,126.2,68.7,68.3,65.0,62.8,61.4,58.8$, 56.6, 55.1, 40.2, 33.5, 30.1, 26.5, 20.1, 19.7; IR (neat) 3273 (br, w), 1600 (w), 1495 (m), $1448(\mathrm{~m}) \mathrm{cm}^{-1}$; MS (EI, $\left.70 \mathrm{eV}\right) \mathrm{m} / z$ (rel int) $263.3\left(4, \mathrm{M}^{+}\right), 179.2(9), 115.1(5), 83.1$ (100); HRMS (EI, $70 \mathrm{eV}$ ) calcd for $\mathrm{C}_{19} \mathrm{H}_{21} \mathrm{~N}\left(\mathrm{M}^{+}\right)$263.1674; found 263.1676.

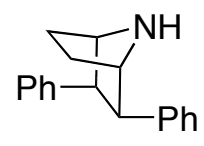

52

$\left(2 R^{*}, 3 R^{*}\right)$-Diphenyl-7-azabicyclo[2.2.1]heptane (52): $n$-Butyllithium $(0.41 \mathrm{~mL}$ of a 2.12 M solution in hexanes, $0.87 \mathrm{mmol}$ ) was added in a dropwise fashion to a solution of stannyl imine 36 (0.26 g, $0.73 \mathrm{mmol})$ and trans-stilbene $(0.30 \mathrm{~g}, 1.6 \mathrm{mmol})$ in THF (2.0 $\mathrm{mL}$ ) cooled to $-78{ }^{\circ} \mathrm{C}$. After $30 \mathrm{~min}$., saturated aqueous ammonium chloride was added and the mixture was diluted with ether then acid/base extracted with $2 \mathrm{M}$ aqueous $\mathrm{HCl}$ and $2 \mathrm{M}$ aqueous $\mathrm{NaOH}$. The combined organic phases were dried $\left(\mathrm{Na}_{2} \mathrm{SO}_{4}\right)$ and concentrated to an oil. Chromatography (10\% methanol/methylene chloride) gave $59 \mathrm{mg}$ (33\%) of $\mathbf{5 2}$ as an oil. Data for $\mathbf{5 2 :} \mathrm{R}_{f}=0.30\left(10 \%\right.$ methanol/methylene chloride); ${ }^{1} \mathrm{H}$ NMR (400 MHz, $\left.\mathrm{CDCl}_{3}\right) \delta$ 7.39-7.17 (m, $\left.10 \mathrm{H}\right), 3.92(\mathrm{t}, 1 \mathrm{H}, J=5.0 \mathrm{~Hz}), 3.80(\mathrm{~d}, 1 \mathrm{H}, J$ $=5.0 \mathrm{~Hz}), 3.46(\mathrm{dd}, 1 \mathrm{H}, J=7.0,5.0 \mathrm{~Hz}), 3.05(\mathrm{~d}, 1 \mathrm{H}, J=7.0 \mathrm{~Hz}), 2.39(\mathrm{br} \mathrm{s}, 1 \mathrm{H}), 1.77-$ $1.68(\mathrm{~m}, 1 \mathrm{H}), 1.64-1.57(\mathrm{~m}, 2 \mathrm{H}), 1.53-1.43(\mathrm{~m}, 1 \mathrm{H}) ;{ }^{13} \mathrm{C} \mathrm{NMR}\left(100 \mathrm{MHz}, \mathrm{CDCl}_{3}\right) \delta$ 146.0, 140.5, 128.7, 128.6, 128.3, 127.2, 126.5, 126.4, 64.4, 62.1, 58.4, 52.3, 31.9, 24.0; IR (neat) 3366 (w), 1603 (m), 1493 (s) $\mathrm{cm}^{-1}$; MS (EI, $\left.70 \mathrm{eV}\right) \mathrm{m} / \mathrm{z}$ (rel int) $249.2\left(2, \mathrm{M}^{+}\right.$), 180.1 (5), 69.1 (100); HRMS (EI, $70 \mathrm{eV}$ ) calcd for $\mathrm{C}_{18} \mathrm{H}_{19} \mathrm{~N}\left(\mathrm{M}^{+}\right)$249.1517; found 249.1509. 


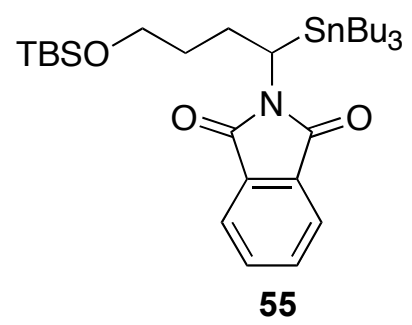

\section{$N$-[4-(tert-Butyldimethylsilyloxy)-1-(tri- $n$-butylstannyl)butyl]phthalimide (55): $n$ -}

Butyllithium (44.0 $\mathrm{mL}$ of a $1.75 \mathrm{M}$ solution in hexanes, $77.0 \mathrm{mmol}$ ) was added to a solution of diisopropylamine $(11.0 \mathrm{~mL}, 7.9 \mathrm{~g}, 78.9 \mathrm{mmol})$ in THF at $0{ }^{\circ} \mathrm{C}$. After $30 \mathrm{~min}$., (tri- $n$-butyl)tin hydride ( $30 \mathrm{~mL}, 32.5 \mathrm{~g}, 112 \mathrm{mmol}$ ) was added in a dropwise fashion to afford a pale green-yellow solution. After $30 \mathrm{~min}$., the solution was cooled to $-78{ }^{\circ} \mathrm{C}$ and 4-(tert-butyldimethylsilyloxy)butyraldehyde (10.0 g, $51.2 \mathrm{mmol})$ in THF $(25 \mathrm{~mL})$ was added. After $20 \mathrm{~min}$. at $-78{ }^{\circ} \mathrm{C}$, saturated aqueous ammonium chloride was added. The mixture was diluted with ether, washed with saturated aqueous ammonium chloride, dried $\left(\mathrm{MgSO}_{4}\right)$, filtered, and concentrated to provide an oil. The crude oil was then dissolved in THF $(200 \mathrm{~mL})$ and cooled to $0{ }^{\circ} \mathrm{C}$. Triphenylphosphine $(14.8 \mathrm{~g}, 56.4$ mmol), phthalimide (8.30 g, $56.4 \mathrm{mmol})$, and diethyl azodicarboxylate $(9.0 \mathrm{~mL}, 57.2$ mmol) were added sequentially. The reaction was allowed to warm to room temperature while stirring overnight. The mixture was concentrated and the residue triturated with hexanes. Concentration of the extracts yielded a yellow oil. Chromatography (0-5\% ethyl acetate/hexanes gradient) afforded $14.2 \mathrm{~g}$ (45\%) of $\mathbf{5 5}$ as a bright yellow oil. Data for 55: $\mathrm{R}_{f}=0.45$ (10\% ethyl acetate/hexanes); ${ }^{1} \mathrm{H}$ NMR (300 MHz, $\left.\mathrm{CDCl}_{3}\right) \delta 7.77$ (ddd, $2 \mathrm{H}, J=5.2,2.9,0.7 \mathrm{~Hz}), 7.65(\mathrm{ddd}, 2 \mathrm{H}, J=5.4,3.2,0.8 \mathrm{~Hz}), 3.91(\mathrm{dd}, 1 \mathrm{H}, J=10.1$, $5.9 \mathrm{~Hz}), 3.60-3.56(\mathrm{~m}, 2 \mathrm{H}), 2.00-1.78(\mathrm{~m}, 2 \mathrm{H}), 1.50-1.39(\mathrm{~m}, 8 \mathrm{H}), 1.26$ (hex, $6 \mathrm{H}, J=$ $7.0 \mathrm{~Hz}), 1.02-0.70(\mathrm{~m}, 24 \mathrm{H}), 0.01(\mathrm{~s}, 3 \mathrm{H}),-0.01(\mathrm{~s}, 3 \mathrm{H}) ;{ }^{13} \mathrm{C} \mathrm{NMR}\left(90 \mathrm{MHz}, \mathrm{CDCl}_{3}\right) \delta$ 
159.1, 133.7, 132.1, 122.8, 62.4, 37.1, 31.4, 29.2, 29.0, 27.4, 25.9, 18.3, 13.6, 10.3, -5.4;

IR (neat) 1772 (w), 1706 (s), 1466 (m), 1388 (m), 1255 (m), 1100 (m); MS (CI, $\left.\mathrm{CH}_{4}\right) \mathrm{m} / \mathrm{z}$ (rel int) 608 (12, M-CH 4 ), 566 (100),1887 (72), 149 (51), 127 (30 ), 99 (39); HRMS (CI, $\mathrm{CH}_{4}$ ) calcd for $\mathrm{C}_{29} \mathrm{H}_{50} \mathrm{NO}_{3} \mathrm{SiSn}\left[\mathrm{M}-\mathrm{CH}_{3}\right]^{+}$608.2582; found 608.2570.

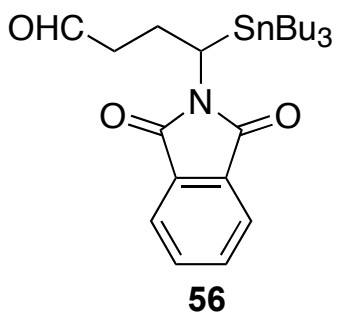

$N$-[4-Oxo-1-(tri-n-butylstannyl)butyl]phthalimide (56): Silane 55 (3.70 g, 5.94 mmol) was dissolved in a 3:1:1 mixture of acetic acid:THF:water $(50 \mathrm{~mL})$. After $15 \mathrm{~h}$, the mixture was diluted with ether, washed with water $(3 \mathrm{x})$, dried $\left(\mathrm{MgSO}_{4}\right)$, filtered, and concentrated to provide an oil. Chromatography (0-20\% ethyl acetate/hexanes gradient) afforded $2.35 \mathrm{~g}(77 \%)$ of the alcohol compound as an oil. Data for $N$-[4-hydroxy-1-(tri$n$-butylstannyl)butyl]phthalimide: $\mathrm{R}_{f}=0.60$ (50\% ethyl acetate/hexanes); ${ }^{1} \mathrm{H}$ NMR (360 $\left.\mathrm{MHz}, \mathrm{CDCl}_{3}\right) \delta 7.78(\mathrm{dd}, 2 \mathrm{H}, J=5.0,3.1 \mathrm{~Hz}), 7.66(\mathrm{dd}, 2 \mathrm{H}, J=5.4,3.0 \mathrm{~Hz}), 3.92(\mathrm{dd}, 1$ $\left.\mathrm{H}, J=9.2,5.8 \mathrm{~Hz},{ }^{2} J\left({ }^{117 / 119} \mathrm{Sn}-{ }^{1} \mathrm{H}\right)=35.5 \mathrm{~Hz}\right), 3.62(\mathrm{t}, 2 \mathrm{H}, J=6.4 \mathrm{~Hz}), 1.53-1.37(\mathrm{~m}, 10$ H) $1.24($ hex, $6 \mathrm{H}, J=7.3 \mathrm{~Hz}), 1.05-0.83(\mathrm{~m}, 15 \mathrm{H}) ;{ }^{13} \mathrm{C} \mathrm{NMR}\left(90 \mathrm{MHz}, \mathrm{CDCl}_{3}\right) \delta 169.2$, 133.8, 132.0, 122.9, 62.3, 36.9, 31.2, 29.2, 28.9, 27.3, 13.6, 10.3; IR (neat) 3447 (br, m), 1703 (s), 1466 (m), 1068 (m); MS (CI, NH${ }_{3}$ ) m/z (rel int) 452 (100, M-C $\left.\mathrm{H}_{4} \mathrm{H}_{9}\right), 235$ (41), 88 (31); HRMS (CI, $\mathrm{NH}_{3}$ ) calcd for $\mathrm{C}_{20} \mathrm{H}_{30} \mathrm{NO}_{3} \mathrm{Sn}\left[\mathrm{M}-\mathrm{C}_{4} \mathrm{H}_{9}\right]^{+} 452.1248$; found 452.1240 . Oxalyl chloride ( $0.34 \mathrm{~mL}, 3.90 \mathrm{mmol})$, dimethyl sulfoxide $(0.55 \mathrm{~mL}, 7.75 \mathrm{mmol}), \mathrm{N}$-[4hydroxy-1-(tri- $n$-butylstannyl)butyl]phthalimide (1.65 g, $3.25 \mathrm{mmol})$, and triethylamine (2.70 mL, $19.3 \mathrm{mmol})$ were sequentially dissolved in $\mathrm{CHCl}_{2}(30 \mathrm{~mL})$ at $-78{ }^{\circ} \mathrm{C}$ over 15 
min. intervals. The reaction was allowed to warm to room temperature. The mixture was diluted with ether, was with saturated aqueous ammonium chloride (5x), dried $\left(\mathrm{Na}_{2} \mathrm{SO}_{4}\right)$, filtered, and concentrated to provide $1.42 \mathrm{~g}(86 \%)$ of $\mathbf{5 6}$ as an oil. The product was used without further purification. Data for 56: $\mathrm{R}_{f}=0.10$ (10\% ethyl acetate/hexanes); ${ }^{1} \mathrm{H}$ NMR (360 MHz, $\left.\mathrm{CDCl}_{3}\right) \delta 9.69(\mathrm{t}, 1 \mathrm{H}, J=1.3 \mathrm{~Hz}), 7.79(\mathrm{dd}, 2 \mathrm{H}, J=5.6,3.1 \mathrm{~Hz}), 7.68$ (dd, $2 \mathrm{H}, J=5.5,3.2 \mathrm{~Hz}), 3.86(\mathrm{dd}, 1 \mathrm{H}, J=11.1,4.6 \mathrm{~Hz}), 2.50-2.36(\mathrm{~m}, 2 \mathrm{H}), 2.30-2.20$ (m, $1 \mathrm{H}), 2.07-1.98(\mathrm{~m}, 1 \mathrm{H}), 1.57-1.38(\mathrm{~m}, 6 \mathrm{H}), 1.31-1.22(\mathrm{~m}, 6 \mathrm{H}), 1.06-0.80(\mathrm{~m}, 15$ $\mathrm{H}) ;{ }^{13} \mathrm{C} \mathrm{NMR}\left(90 \mathrm{MHz}, \mathrm{CDCl}_{3}\right) \delta 201.3,169.1,133.9,131.8,123.0,42.8,36.4,28.9$, 27.3, 25.3, 13.5, 10.4; IR (neat) 1703 (s), 1466 (m), 1388 (s), 1334 (m), 1072 (m); MS (CI, $\left.\mathrm{NH}_{3}\right) \mathrm{m} / z$ (rel int) 507 (18,M+H), 450 (100), 308 (31), 235 (24), 217 (29), 147 (20); HRMS (CI, $\mathrm{NH}_{3}$ ) calcd for $\mathrm{C}_{20} \mathrm{H}_{28} \mathrm{NO}_{3} \mathrm{Sn}\left[\mathrm{M}-\mathrm{C}_{4} \mathrm{H}_{9}\right]^{+}$450.1078; found 450.1091.

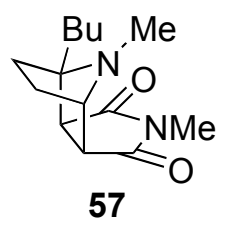

$\left(1 R^{*}, 2 S^{*}, 6 R^{*}\right)-1-B u t y l-4,10$-dimethyl-4,10-diazatricyclo[5.2.1.0 $\left.{ }^{2.6}\right]$ decane-3,5-dione (57): Stannane $21(82 \mathrm{mg}, 0.20 \mathrm{mmol})$, methyl iodide (60 $\mu \mathrm{L}, 137 \mathrm{mg}, 1.0 \mathrm{mmol}), N$ methylmaleimide (44 mg, $0.4 \mathrm{mmol})$ were dissolved in toluene $(2 \mathrm{~mL})$ and heated to 110 ${ }^{\circ} \mathrm{C}$ for $18 \mathrm{~h}$ in a sealed tube. The reaction mixture was diluted with methylene chloride and washed with water $(1 \mathrm{x})$. The combined organic phases were dried $\left(\mathrm{Na}_{2} \mathrm{SO}_{4}\right)$ and concentrated to an oily solid. Chromatography ( $0-1 \%$ methanol: methylene chloride gradient) gave $20 \mathrm{mg}(40 \%)$ of $\mathbf{5 7}$ as a solid off-white powder. Data for 57: $\mathbf{R}_{f}=0.11$ (2\% methanol: methylene chloride); $\mathrm{mp}=86-90{ }^{\circ} \mathrm{C} ;{ }^{1} \mathrm{H}$ NMR $\left(500 \mathrm{MHz}, \mathrm{CDCl}_{3}\right) \delta 3.55$ (d, $1 \mathrm{H}, J=4.5 \mathrm{~Hz}), 2.94(\mathrm{~s}, 3 \mathrm{H}), 2.67(\mathrm{~d}, 1 \mathrm{H}, J=7.0 \mathrm{~Hz}), 2.58(\mathrm{~d}, 1 \mathrm{H}, J=7.0 \mathrm{~Hz})$, 2.01 (s, 3 H), 2.00-1.96 (m, 1 H), 1.82-1.77 (m, 2 H), 1.56-1.38 (m, 7 H), 0.97 (t, 3 H, $J=$ 
$7.5 \mathrm{~Hz}) ;{ }^{13} \mathrm{C} \mathrm{NMR}\left(125 \mathrm{MHz}, \mathrm{CDCl}_{3}\right) \delta 178.9,177.1,71.4,65.1,50.7,48.8,31.8,30.8$, 30.0, 28.1, 26.6, 25.0, 23.7, 14.3; IR $\left(\mathrm{CHCl}_{3}\right) 1696$ (s), 1435 (m), 1287 (m) $\mathrm{cm}^{-1}$; MS (EI, $70 \mathrm{eV}) \mathrm{m} / \mathrm{z}$ (rel int) $250.3\left(13, \mathrm{M}^{+}\right), 221.2$ (26), 208.2 (52), 179.2 (51), 139.2 (52), 96.1 (100); HRMS (EI, $70 \mathrm{eV}$ ) calcd for $\mathrm{C}_{14} \mathrm{H}_{22} \mathrm{~N}_{2} \mathrm{O}_{2}\left(\mathrm{M}^{+}\right)$250.1681; found 250.1682.

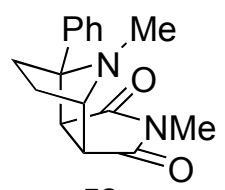

58

$\left(1 R^{*}, 2 R^{*}, 6 S^{*}\right)-4,10$-Dimethyl-1-phenyl-4,10-diazatricylo[5.2.1.0 $\left.{ }^{2.6}\right]$ decane-3,5-dione

(58): Stannane 19 (43 mg, $0.10 \mathrm{mmol}$ ), methyl iodide ( $30 \mu \mathrm{L}, 68 \mathrm{mg}, 0.5 \mathrm{mmol}), N$ methylmaleimide (17 mg, $0.15 \mathrm{mmol})$ were dissolved in toluene $(1 \mathrm{~mL})$ and heated to $110{ }^{\circ} \mathrm{C}$ for $18 \mathrm{~h}$ in a sealed tube. The reaction mixture was diluted with methylene chloride and washed with water $(1 \mathrm{x})$. The combined organic phases were dried $\left(\mathrm{Na}_{2} \mathrm{SO}_{4}\right)$ and concentrated to an oily solid. Chromatography (0-1\% methanol: methylene chloride gradient) gave $5.0 \mathrm{mg}(19 \%)$ of $\mathbf{5 8}$ as a solid off-white film. Data for $\mathbf{5 8}: \mathbf{R}_{f}=0.20$ (2\% methanol:methylene chloride); ${ }^{1} \mathrm{H}$ NMR (500 MHz, $\left.\mathrm{CDCl}_{3}\right) \delta$ 7.40-7.20 (m, $\left.5 \mathrm{H}\right), 3.81$ $(\mathrm{d}, 1 \mathrm{H}, J=4.5 \mathrm{~Hz}), 2.96(\mathrm{~s}, 3 \mathrm{H}), 2.76(\mathrm{q}, 2 \mathrm{H}, J=7.0 \mathrm{~Hz}), 2.24-2.18(\mathrm{~m}, 1 \mathrm{H}), 2.07(\mathrm{dd}$, $2 \mathrm{H}, J=8.0,5.5 \mathrm{~Hz}), 2.02(\mathrm{~s}, 3 \mathrm{H}), 1.72-1.67(\mathrm{~m}, 1 \mathrm{H}) ;{ }^{13} \mathrm{C} \mathrm{NMR}\left(125 \mathrm{MHz}, \mathrm{CDCl}_{3}\right) \delta$ $178.4,176.1,137.1,128.6,128.2,127.8,74.9,64.9,56.1,49.5,32.3,30.9,26.4,25.1 ;$ IR $\left(\mathrm{CHCl}_{3}\right) 1696(\mathrm{~s}), 1444(\mathrm{~m}), 1290(\mathrm{~m}) \mathrm{cm}^{-1}$; MS (EI, $\left.70 \mathrm{eV}\right) \mathrm{m} / \mathrm{z}$ (rel int) $270.2\left(58, \mathrm{M}^{+}\right)$, 242.2 (55), 184.2 (32), 159.2 (100), 118.1 (35); HRMS (EI, 70eV) calcd for $\mathrm{C}_{16} \mathrm{H}_{18} \mathrm{~N}_{2} \mathrm{O}_{2}$ $\left(\mathrm{M}^{+}\right)$270.1368; found 270.1358. 